\title{
INTERNAL CONSTITUTION AND THERMAL HISTORIES OF THE TERRESTRIAL PLANETS
}

Hall: On behalf of the Organizing Committee of Joint Discussion A it gives me great pleasure to introduce our next speaker, Dr B. Levin. He is Chief of the Department on the Evolution of the Earth at the Schmidt Institute of Physics of the Earth at Moscow. He will present an invited paper on the Internal Constitution and Thermal Histories of Terrestrial Planets.

Levin: The problems of the internal constitution of the terrestrial planets and that of their thermal histories are closely connected. We are interested not only in giving the distribution of density in planetary interiors and their stratification into physically distinct layers, but also in giving some insight into the chemical gross composition of planets. At the same time the thermal histories of the terrestrial planets strongly depend on the content of radioactive elements and thus on the chemical composition. The differentiation of planetary interiors into chemically distinct layers depends on their temperatures which determine their viscosity, but, on the other hand, the redistribution of radioactive elements in the course of differentiation seriously change the further course of the thermal history. The controversial problem of thermal convection in planetary interiors depends both on their constitution and on their thermal history.

Even for the Earth, we have no unique solution for the present day constitution and present day thermal state of its interior. The radial distribution of density is rather well known, but our knowledge of radial changes in composition is insufficient. Especially vague is our knowledge of the composition of both the outer and inner parts of the Earth's core. The uncertainty of the thermal history of the Earth depends mainly on a poor knowledge of the initial temperature and of the dependence of the thermal properties on pressure. The estimates of the initial temperature of the Earth and of its content of radioactive elements are based on cosmogonical ideas on the formation process of the Earth. For other terrestrial planets the observational data are fewer and the role of cosmogonical ideas is larger.

For all bodies of the terrestrial group, except the Moon, the role of the temperature of their interiors can be neglected as compared with the role of the pressure. For them the radial distribution of density can be computed from the equation of hydrostatic equilibrium using the pressure-density relations for suggested planetary material.

A decade ago the values of the masses and radii of planets necessary for the calculations were poorly determined for some planets. As a result different authors using different numerical values reached conflicting conclusions. At the present time the remaining uncertainty in masses and radii is not serious but other ambiguities remain and lead to discordant results. 
In most calculations of planetary models, the Earth is used as a prototype. The probable radial gradient of composition of the upper mantle produced by the formation of the crust is neglected and the Earth is regarded as being composed of several chemically homogeneous layers. Then the change of density versus depth can be transferred into the change of density versus pressure for the material of a given layer (see Levin, 1970). Or one can start from a practically linear dependence of compressibility on pressure (Lyttleton, 1963). Another way is to start from laboratory data on compressibilities of different minerals or oxides. (Binder, 1969; Reynolds and Summers, 1969).

Differences in pressure-density relations except these connected with suggested phase changes are not important for the final conclusions obtained by different authors.

An important controversial problem is that of the fractionation of iron. Different groups of chondritic meteorites contain from 20 to $30 \%$ of iron in the form of iron oxides in silicates or as metallic nickel-iron, or both. It is widely accepted that these differences represent the corresponding differences of iron-content in meteorite parent bodies and therefore they manifest some fractionation of iron in the asteroidal zone where these bodies formed. However, it is not definitely excluded that they represent differences in the composition of different layers of the parent bodies.

The terrestrial planets as a group, including Mercury, show a much more pronounced fractionation of iron, demonstrated by a low density of the Moon and a high density of Mercury. The only reasonable way to explain Mercury's density of $5.5 \mathrm{~g} / \mathrm{cm}^{3}$ is to accept that it contains $60-70 \%$ of metallic nickel-iron. (Plagemann, 1965; Kozlovskaya, 1969; Reynolds and Summers, 1969). On the other hand, the low density of the Moon and its moment-of-inertia which is very close to 0.4 , indicate that the Moon contains no, or almost no, metallic iron. (Kozlovskaya, 1962; Levin, 1966b, Nakamura and Latham, 1969). The iron-content of lunar silicates can be about $20-25 \%$, and thus its total iron content can be close to that of chondritic meteorites.

To explain the high density of Mercury it is not sufficient to suppose that all iron contained in a chondrite-type material is in Mercury in a metallic state. Mercury must be strongly enriched in iron, as compared with chondrites.

To explain the great abundance of iron in Mercury we must assume either that magnetic properties of iron particles may have caused their preferential agglomeration, or that silicates particles were preferentially eliminated.

To explain the metallic state of iron in Mercury we can refer to the planet's proximity to the Sun and assume that it accumulated from the dust which never cooled below $400 \mathrm{~K}$ and that therefore the iron particles that condensed in the cooling solar nebula, remained unoxidized by water vapour.

The degree of fractionation for the remaining bodies of the terrestrial group depends on the nature of dense cores in the Earth and Venus.

For Mars the situation is now quite clear. A few years ago some authors adopted for Mars too low a value for its radius and therefore too high a value for its density. That permitted them to regard this planet as containing an iron core of the same relative mass as that of the Earth. Now it is firmly established that Mars' radius is almost 
$3400 \mathrm{~km}$ and its density is about $3.9 \mathrm{~g} / \mathrm{cm}^{3}$. Mars must have an iron core but it comprises no more than $5-10 \%$ of the total mass. It is at least 3 times smaller than the Earth's core. (MacDonald, 1962; Kozlovskaya, 1966; Binder, 1969; Reynolds and Summers, 1969). The calculated size of the Martian core depends not only on the adopted pressure-density relation for the Martian mantle, but also on the adopted thickness of a Martian crust composed of light silicates. Calculations of the thermal history of Mars show that its interior probably was at some time molten throughout and therefore the thickness of the light crust can be up to 100 or even $200 \mathrm{~km}$.

For the Earth and Venus there is no doubt that they both contain dense cores but two hypotheses about their nature exist: the old iron-core hypothesis and the hypothesis of metallized silicates.

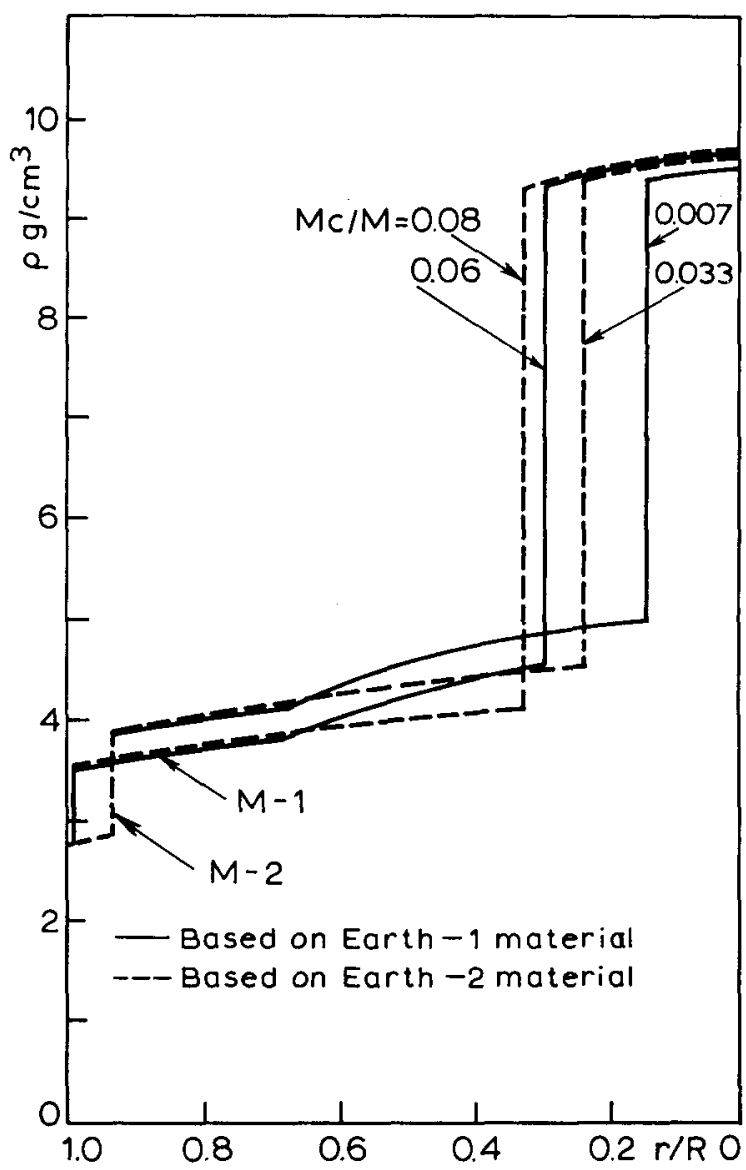

Fig. 1. Mars: Radial density distribution for 4 models with moment of inertia as determined from observations. $\left[M_{\mathrm{c}} / M\right.$ - relative mass of the iron core; $\mathrm{M}-1$ - models with assumed thickness of the outer light crust $\left(\varrho=2.8 \mathrm{~g} / \mathrm{cm}^{3}\right)$ equal to $20 \mathrm{~km} ; \mathrm{M}-2$ - models with a crust of $216 \mathrm{~km}$; Earth-1, Earth-2 - correspond to terrestrial models with mantle densities of 3.32 and $3.60 \mathrm{~g} / \mathrm{cm}^{3}$ at the boundary with the crust]. 
For a long time the dense core of the Earth was believed to be composed of nickeliron, similar to that in meteorites. But a decade ago, experiments on shock compression of iron up to pressures of the millions of atmospheres existing in the Earth's core, showed that under these pressures the density of nickel-iron is greater than that of the material of the core deduced from geophysical data. (Al'tshuler et al., 1958; McQueen and Marsh, 1960; Zharkov, 1960, 1962.) Since then a modified form of the iron-core hypothesis is adopted by geophysicists : they assume that the Earth's core is composed of an iron-rich alloy containing nickel, and also silicon, or carbon, or something else to decrease its density. (MacDonald and Knopoff, 1958; Kormer and Funtikov, 1965). Only the small inner core comprising about $2 \frac{1}{2} \%$ of the Earth's mass can be composed of nickel-iron.

Even before this modification of the iron-core hypothesis, there were difficulties with the explanation of the origin of metallic nickel-iron in the material of the Earth, with its sedimentation into the core, and with other questions. For the modified form of the hypothesis these difficulties became much more serious (Levin, 1970). We will return to them somewhat later, after discussing the alternative hypothesis of metallized silicates.

In this hypothesis it is assumed that the jump in density at the core-mantle boundary is caused by a pressure-induced transition of silicates into a dense metallic state. The pressure of about one and a half million atmospheres existing at the core-mantle boundary is regarded as a critical pressure for this transition. The inner core of the Earth can again be regarded as composed of nickel-iron, perhaps segregated not from the whole Earth but only from the metallized liquid part of the core.

The origin of the core of metallized silicates presents no difficulties: it had to appear when the growing Earth reached about 0.8 of its present mass and the central pressure reached the critical value. The further growth of the Earth increased the size of the core up to its present size.

For metallized silicates the melting temperature is much lower than for iron or for non-metallized silicates in the lower mantle. Therefore it is easy to combine the liquid state of the outer core with the solid state of the mantle which is almost impossible on the basis of the iron-core hypothesis.

The metallized silicates must contain as much radioactive material as 'normal' silicates. Their heat generation permits us to explain convective currents in the core, which are usually regarded as the source of the Earth's magnetic field. In an iron-rich alloy the content of radioactive elements must be very small - of the same order of magnitude as in iron meteorites - and the motions in the core must be ascribed to some other source of energy.

And last but not least - the hypothesis of metallized silicates eliminates the necessity for the large and puzzling difference in composition between the Earth and the Moon which forms a cosmochemical basis for the hypothesis that the Moon was captured by the Earth.

Unfortunately the hypothesis of metallized silicates has a major shortcoming: it has been proved neither by calculation nor by experiment. 
The jump in density at the core-mantle boundary is so large that it cannot be ascribed to some changes in electronic shells of metallic ions in silicates because they occupy only $10 \%$ of the volume. There must be changes in the electronic shells of oxygen which occupy $90 \%$ of the volume. But electronic shells of oxygen are very stable. Although they have been unable to make exact calculations, theoretical phy. sicists nevertheless suggest that any changes in these shells require a much larger pressure than that existing at the core-mantle boundary.

Experiments on shock-compression of rock-samples have failed to detect the necessary phase transition although several samples were compressed at more than 2 million atmospheres and one sample even up to 5 million atmospheres. (Trunin et al., 1965). However, substances exist that under shock compression require much larger pressures than equilibrium ones for phase transition. Therefore, in spite of the negative result of experiments it is premature to reject the hypothesis of metallized silicates.

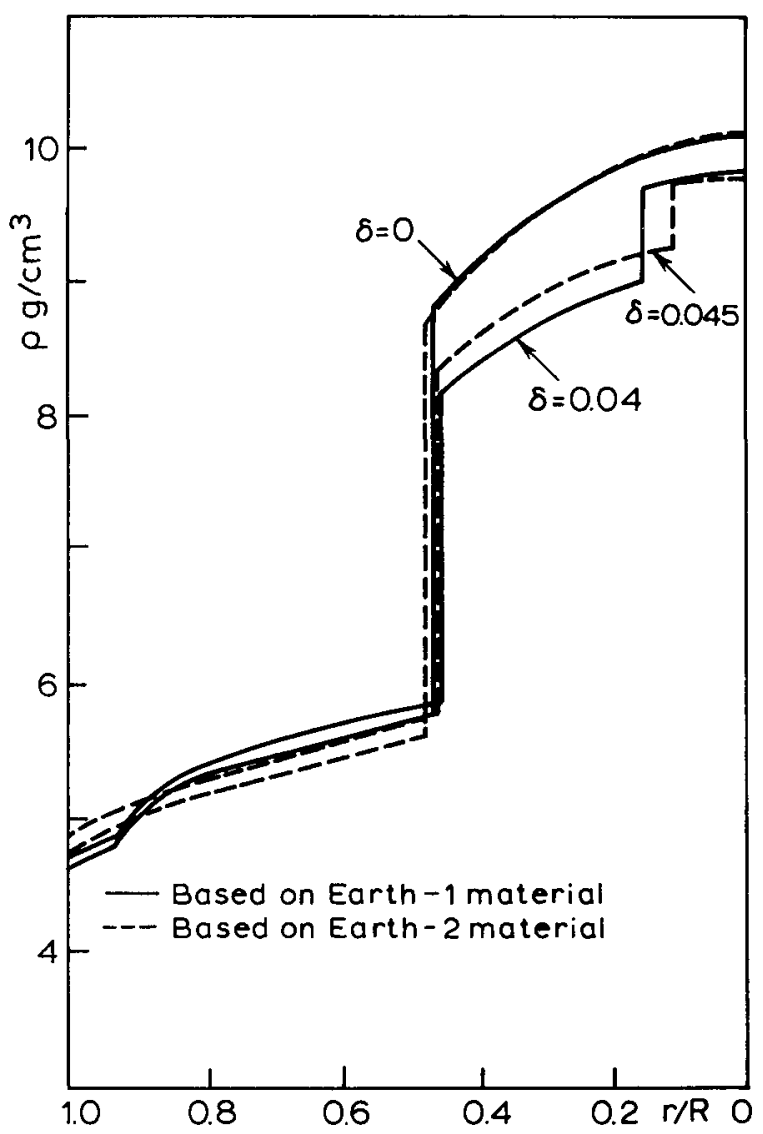

Fig. 2. Venus: density distribution along the radius for 4 models. Two models of material with pressure-density relation multiplied by a constant factor $1+\delta$ contain, besides the iron core, a layer of metallized silicates. 
If we accept this hypothesis for the outer core of the Earth, we can accept it also for the core of Venus. Similar to that of the Earth, the core of Venus can contain an outer part of metallized silicates and an inner core of nickel-iron. (Kozlovskaya, 1966; Levin, 1970).

On the basis of the hypothesis of metallized silicates the iron content calculated for terrestrial planets, excluding Mercury, and for the Moon varies from 20 to $30 \%$, in the same range as that of the different groups of chondritic meteorites. On the ironcore hypothesis, variations are much larger - from 20 to $40-50 \%$. Some authors believe that a correlation exists with the distance from the Sun; but the iron-content in Venus is smaller than in the Earth and the Moon must be totally excluded.

Let us return to some difficulties of the iron core hypothesis. In spite of the recent modification of iron core hypothesis many authors continue to discuss the formation of a core of nickel-iron, not of some lighter iron-rich alloy. Usually they simply suggest that the material from which the terrestrial planets were accumulated contained metallic nickel-iron in addition to silicates. Most authors start from the premise that the Earth core was formed after the accumulation of the Earth. To make possible the segregation of nickel-iron into the core, one assumes following Elsässer (1963) that the process of core formation had to begin when the temperature of the outer layers reached the melting temperature. The further release of gravitational potential energy in the course of the settling of iron could melt the whole interior of the Earth, permitting the formation of the core.

To provide for this melting Hanks and Anderson (1969) arbitrarily increase the accumulation rate and thus the intensity of bombardment, so that the whole accumulation takes only $2 \times 10^{5}$ yr or even less.

This idea of a rapid accumulation of the Earth became very popular. Unfortunately it is not correct and probably even not necessary to achieve partial melting. According to calculations by Safronov $(1954,1958,1969)$ and Urey (1969) the accumulation time must be of the order of $10^{8} \mathrm{yr}$ for a mass of the solar nebula of about $5-10 \%$ of the solar mass. The accumulation of the Earth could be more rapid in a much more massive nebula suggested by Cameron (1962). But in such a case in the Earth's zone of the nebula a number of bodies of asteroidal size or larger composed of nonvolatile substances should be left after the Earth reached its present mass, and it seems to me that it is impossible to get rid of them.

In 1965 Safronov showed that a high initial temperature of the Earth is inevitable even for a slow rate of accumulation, if one takes into account that a large fraction of the total mass was brought by asteroidal sized bodies. While impacts of dust particles release heat at the very surface so that it is easily radiated into space, impacts of asteroidal sized bodies release a few percent of the energy in the form of seismic waves, which heat the deep interior. This leads to a much higher initial temperature for the Earth than that calculated from the impact of dust particles.

The energy distribution at such crater forming impacts is not well known. Nevertheless if we really need to melt the layers of the Earth at the depth of $200-500 \mathrm{~km}$ it can be explained with a much smaller reduction of the accumulation time. 
The heat released during the core formation is sufficient to increase the average temperature of the Earth by about $2000^{\circ} \mathrm{C}$. (Lustikh, 1948; Urey, 1952; Birch, 1965). The analysis of this process shows that after the formation of the iron core the whole mantle would be at the temperature at which melting begins. But the further release of radiogenic heat would melt the mantle (Majeva, 1971a). However, we have seismic evidence that it is solid. The only possibility for obtaining a solid mantle is to accept thermal convection in the mantle.

The possibility of convection was studied by several authors for more and more

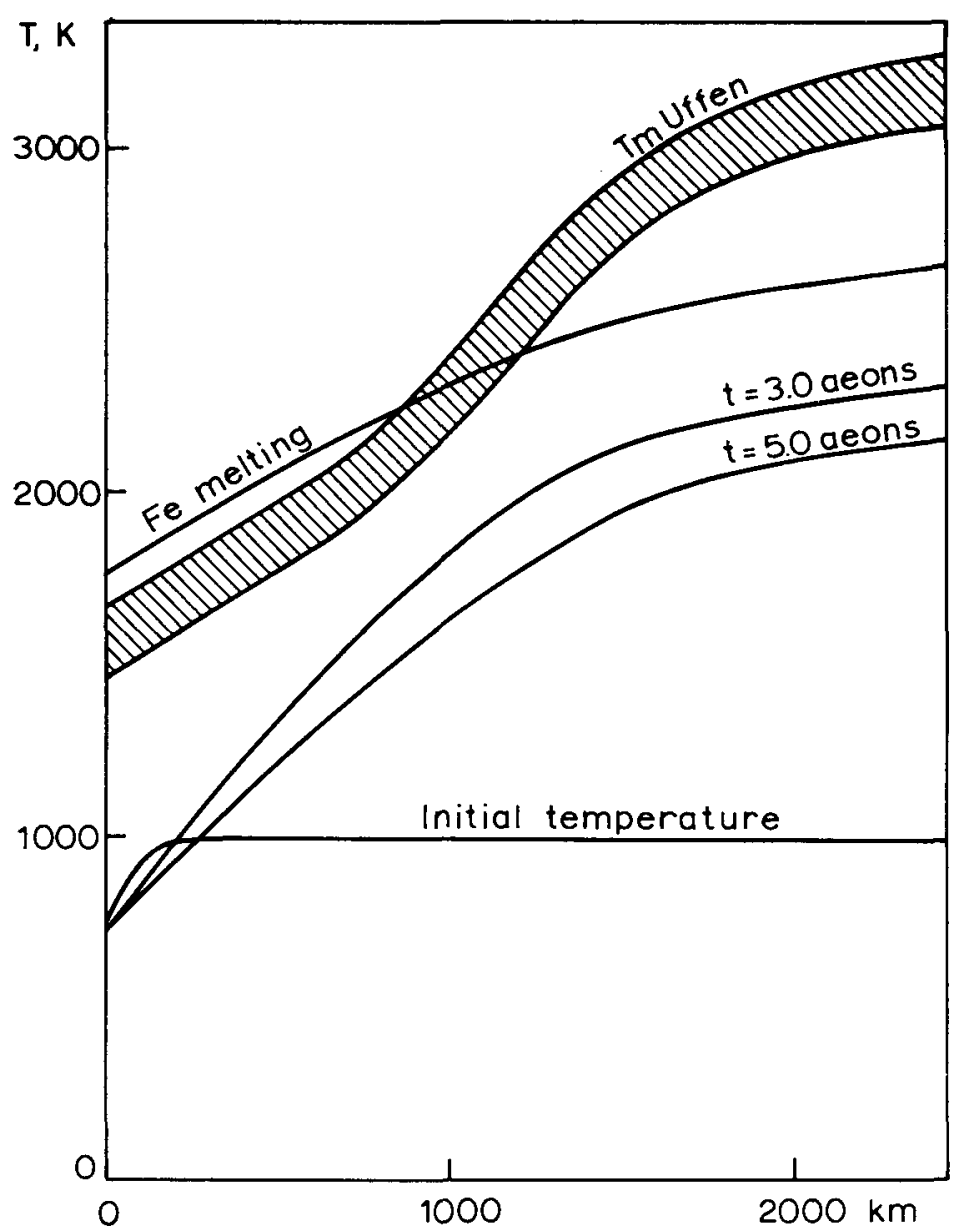

Fig. 3. Mercury: calculated distribution of temperature for different moments of time for a model in which $60 \%$ of metallic iron containing no radioactive elements is uniformly mixed with silicates containing U: $2 \times 10^{-8} \mathrm{~g} / \mathrm{g}$; Th: $8 \times 10^{-8} \mathrm{~g} / \mathrm{g} ; \mathrm{K}: 8 \times 10^{-4} \mathrm{~g} / \mathrm{g}$. The assumed initial distribution of temperature corresponds to the moment of time $t=0.2 \times 10^{9} \mathrm{yr}$ after the beginning of accumulation of the planet. The hatched zone represents the temperature interval for melting of silicates. Its upper boundary corresponds to melting temperature of terrestrial silicates after Uffen (1952) recalculated for pressures inside Mercury. 
complicated models of planetary interiors. The answer was always positive. For example, Schubert et al. (1969) have demonstrated the instability of planetary interiors for a model including an increase of viscosity with depth and internal heat generation. However, in all these studies the chemical homogeneity of the convecting medium is implicitly assumed. But such homogeneity is highly improbable if the mantle of the Earth (and of the other planets) has undergone a differentiation, at least one that formed a crust.

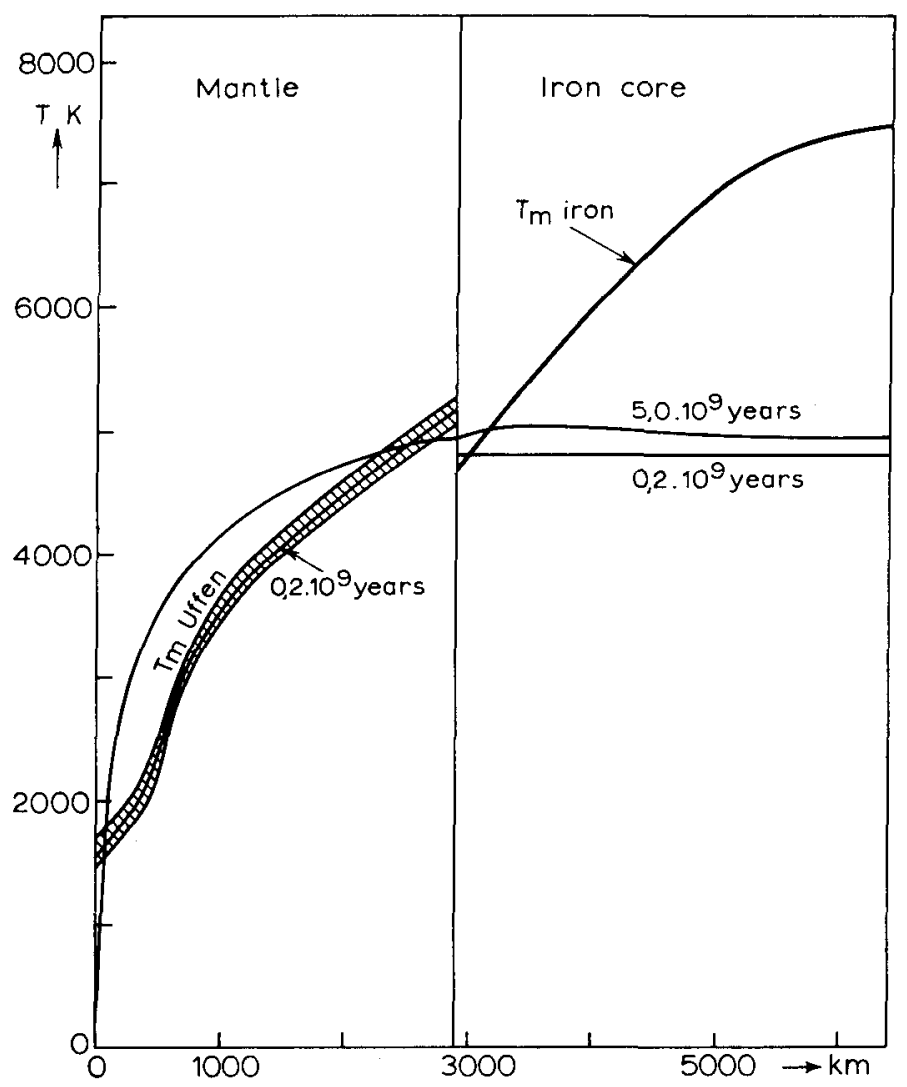

Fig. 4. Earth: initial $\left(t=0.2 \times 10^{5} \mathrm{yr}\right)$ and present-day $\left(t=5 \times 10^{5} \mathrm{yr}\right)$ distributions of temperature in a model with iron core formed after the accumulation of the Earth. The 'initial' distribution of temperature was calculated assuming that the gravitational energy released during the setting of iron into core heated the whole mantle up to the same semi-molten state. The initial heating of the iron core nearly to the melting temperature of silicates at the core-mantle boundary absorbed about

$20 \%$ of the released heat. (For explanation of the hatched zone, see caption to Figure 3.)

On the other hand, new evidence obtained during recent studies of the ocean floor is interpreted by many geophysicists as due to mantle convection. But serious contradictions exist and this interpretation is not generally accepted.

There is another objection against the formation of the core by the settling of iron: 
if the whole mantle were once molten, the differentiation of silicates would produce a much thicker crust than the actual one. Also, the formation of the crust would be a rather rapid process confined to the early history of the Earth while there is geological and geochemical evidence that it was a slow process continuing up to the present time.

The attempts to visualize the core formation as described above explain the core of nickel iron, but not the iron rich alloy containing also silicon or carbon.

The only attempt to justify a core containing silicon was made by Ringwood (1959, 1966, 1970). He suggests that the Earth accumulated from material similar to carbonaceous chondrites, containing only oxidized iron.

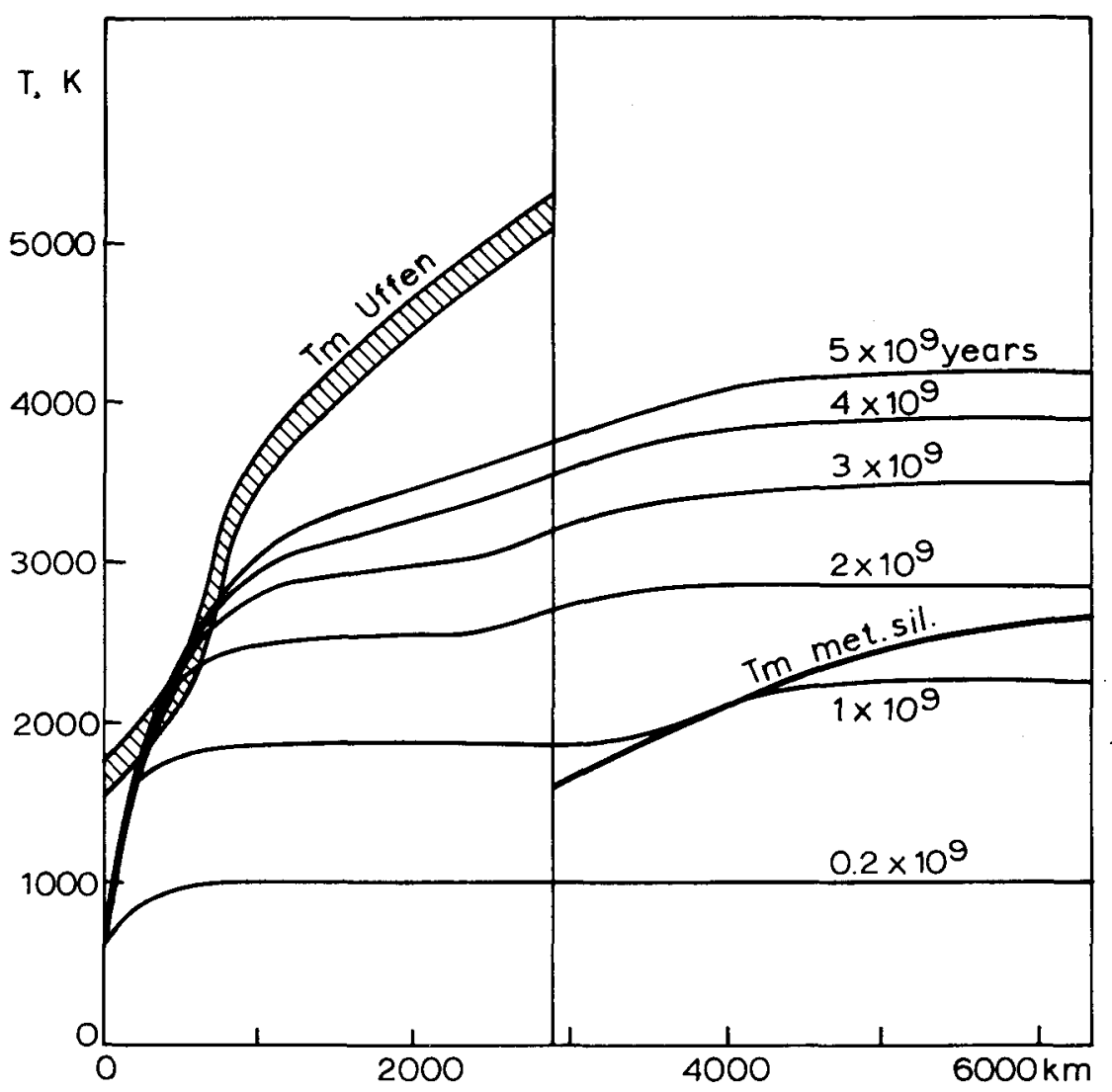

Fig. 5. Earth: distribution of temperature for different moments of time in a model with a core of metallized silicates $\left(\mathrm{U}: 2 \times 10^{-8} \mathrm{~g} / \mathrm{g} ;\right.$ Th:8 $\times 10^{-8} \mathrm{~g} / \mathrm{g} ; \mathrm{K}: 8 \times 10^{-4} \mathrm{~g} / \mathrm{g}$; heat capacity $C_{\text {martle: }}$ : $0.3 \mathrm{cal} / \mathrm{g} \mathrm{grad} ; C_{\text {core }}: 0.2 \mathrm{cal} / \mathrm{g} \mathrm{grad}$ ). (For explanation of the hatched zone, see caption to Figure 3.)

Ringwood suggests that at the end of the accumulation the temperature of the outer layers of the Earth was so high, due to intense bombardment and an extensive insulating atmosphere, that not only iron and nickel but also silicon were reduced by carbon and settled into the core. As ferrosilicium is out of equilibrium with silicates, 
Ringwood assumes that the sinking lumps were sufficiently large to retain silicon.

However, even the intense solar wind probably emitted by the young Sun scarcely could blow off the enormous quantity of carbondioxide which had to be formed in this reduction process.

The course of accumulation in the zone of the terrestrial planets should also be different from that suggested by Ringwood. The dust component of the solar nebula rapidly accumulated into a multitude of asteroidal size bodies but their later accumulation into the Earth and planets lasted about $10^{8} \mathrm{yr}$. At the end of this process the intensity of bombardment by these bodies (and by their fragments) gradually decreased due to the exhaustion of accretable material.

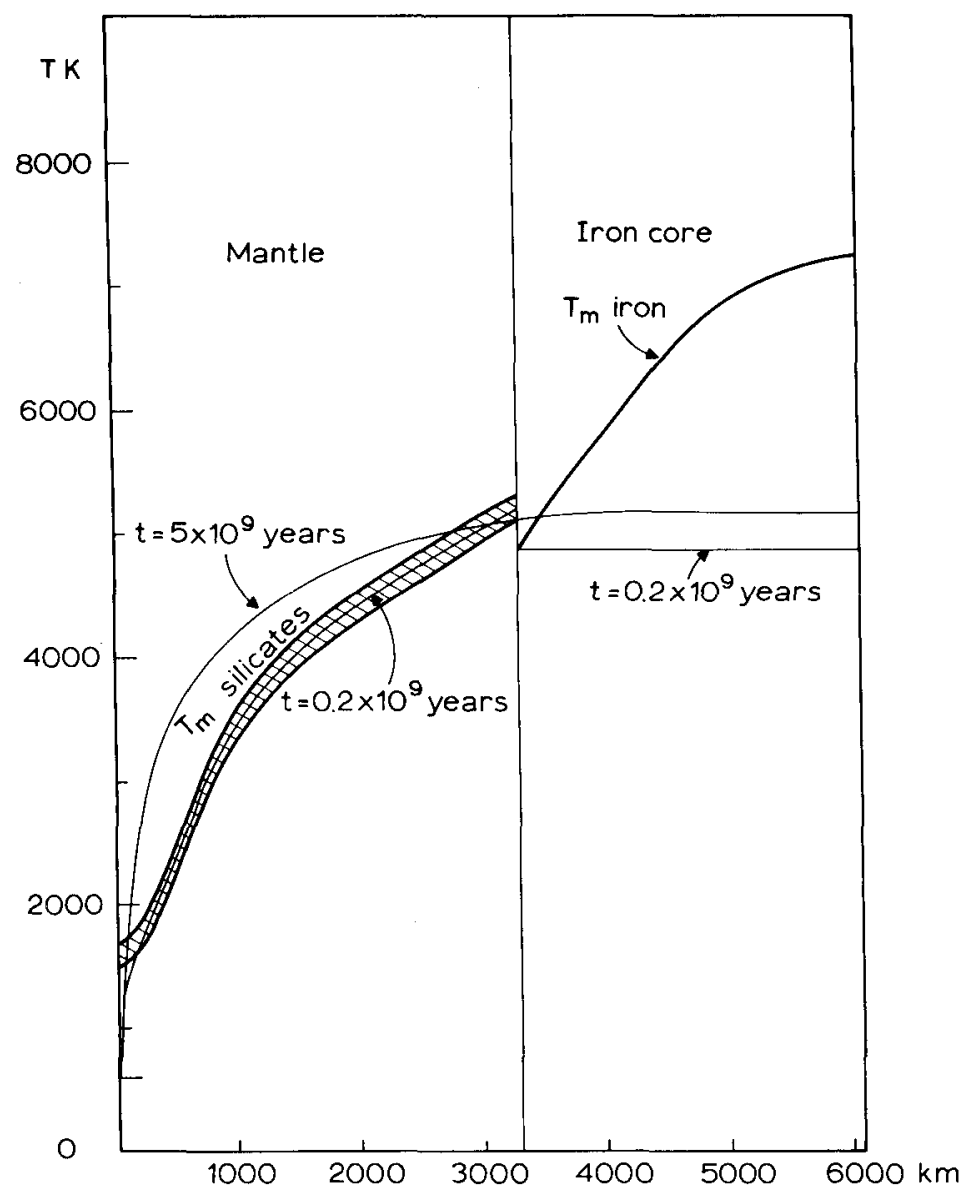

Fig. 6. Venus: initial ( $t=0.2 \times 10^{9} \mathrm{yr}$ ) and present-day $\left(t=5 \times 10^{9} \mathrm{yr}\right.$ ) distribution of temperature in a model with iron core formed after the accumulation of Venus. As for the Earth (Figure 4), the 'initial' distribution of temperature was calculated assuming that the gravitational energy released during the setting of iron into core heated the whole mantle up to the same semimolten state. The initial heating of the iron core nearly up to the melting temperature of silicates at the core-mantle boundary absorbed about $15 \%$ of the released heat. 
Again the melting of the whole mantle is difficult to reconcile with its present day solid state and with the small thickness of the crust. The initial inhomogeneity of terrestrial interiors, suggested by Ringwood, can lead to gravitational differentiation and overturn of layers of different density, but it cannot lead to the homogeneous interiors necessary for thermal convection.

During the last decade the study of element fractionation in meteorites and of the condensation of dust in the cooling solar nebula led Larimer and Anders (1967) to conclude that the accumulation of the meteorite parent bodies possibly occurred, or

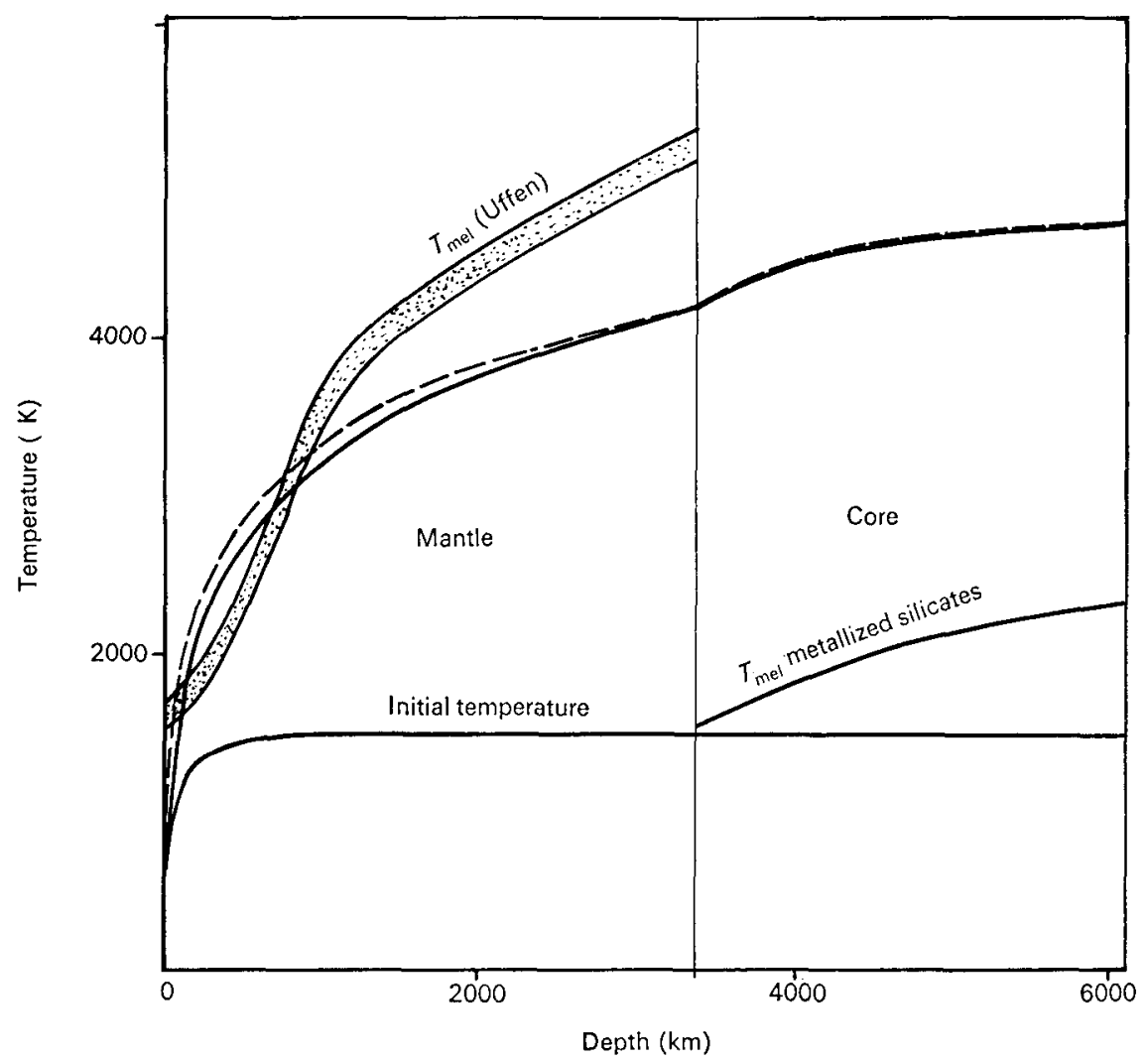

Fig. 7. Venus: temperature distribution for different moments of time in a model with a core of metallized silicates (a model similar to that of Earth, Figure 5).

at least began, during the condensation process. A year ago this hypothesis was applied by Turekian and Clark (1969) to the accumulation of the Earth. A few months ago a study of element fractionation in lunar samples permitted Anders and his coworkers (Ganapathy et al., 1970) to apply it also to the accumulation of the Moon.

I am unable to discuss in details the cosmochemical aspects of this hypothesis and will restrict myself to its main theme. 
In a cooling nebula of solar composition metallic iron condenses when the temperature drops to about $1300 \mathrm{~K}$ while most silicates condense at about $1000 \mathrm{~K}$. (Larimer, 1967). It is assumed that metallic iron particles were able to accumulate into a big body - a future iron core of the Earth - before the decrease of temperature permitted the condensation of silicates. Later the silicate particles had to accumulate around the iron core to form a mantle of the Earth.

As you see, this hypothesis again considers the formation of a nickel iron core, but not of a core of some lighter iron-rich alloy required by modern data.

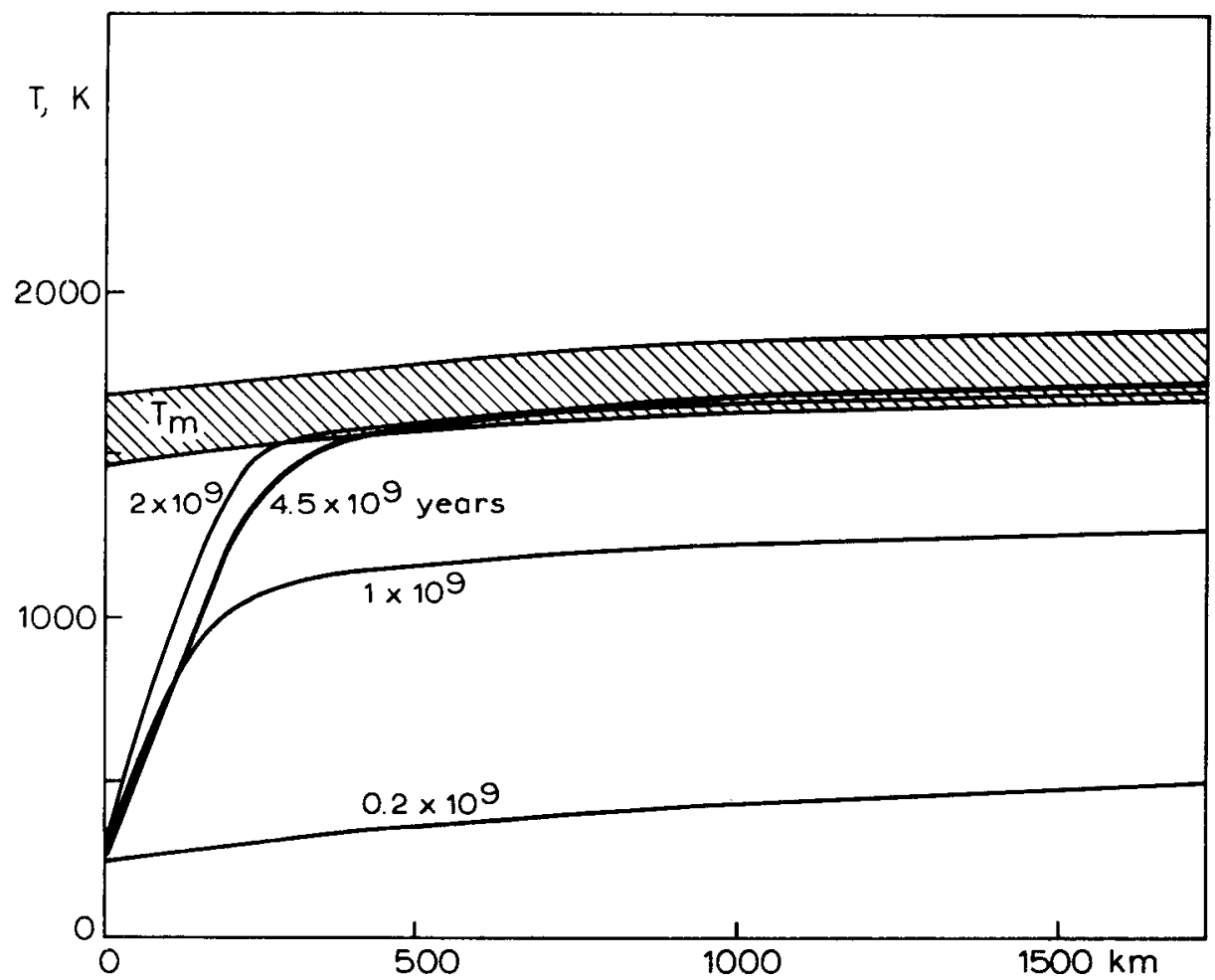

Fig. 8. Moon: temperature distribution for different moments of time in the equatorial zone. (After partial melting $95 \%$ of radioactive elements are transferred to the surface.) The hatched zone represents the assumed temperature interval for melting of silicates. Its upper boundary corresponds to melting of dunite.

The first shortcoming of this hypothesis is the assumption that it is possible to accumulate quickly all metallic particles into a single body. Really a multitude of asteroidal sized bodies could be quickly formed, but their further accumulation into a single body is a very slow process requiring about $10^{8} \mathrm{yr}$. By that time silicates must be condensed and accumulated together with the metallic bodies (planetesimals). Otherwise the cooling time of the nebula (in the terrestrial zone) should be larger than the accumulation time. It seems to be scarcely possible to decrease the existing estima- 
tes of accumulation time by 3 orders of magnitude. To increase the cooling time of the nebula up to $10^{8} \mathrm{yr}$ requires a similar duration of the recession of the nebula from the vicinity of the Sun or a similar duration of the high luminosity stage of the Sun. Both these possibilities seem to be improbable.

In summing up, I would like to stress that we still have no satisfactory explanation of the formation process of the Earth's core on the basis of the iron-core hypothesis. It is so even for a nickel-iron core, not to say of the core of some lighter iron-rich alloy. Therefore we must continue to consider favourably the hypothesis of metallized silicates. Difficulties in proving the reality of pressure induced phase transitions in silicates are not more serious than those in explaining the formation of the iron core in the Earth. The advantages of the hypothesis of metallized silicates in the explanation of some modern properties of the Earth (liquid state of the outer core, convection in it, etc.) are very significant.

The time is short and I will present only a few additional remarks about the thermal history of the terrestrial planets.

During the last two decades several authors have published calculations of thermal

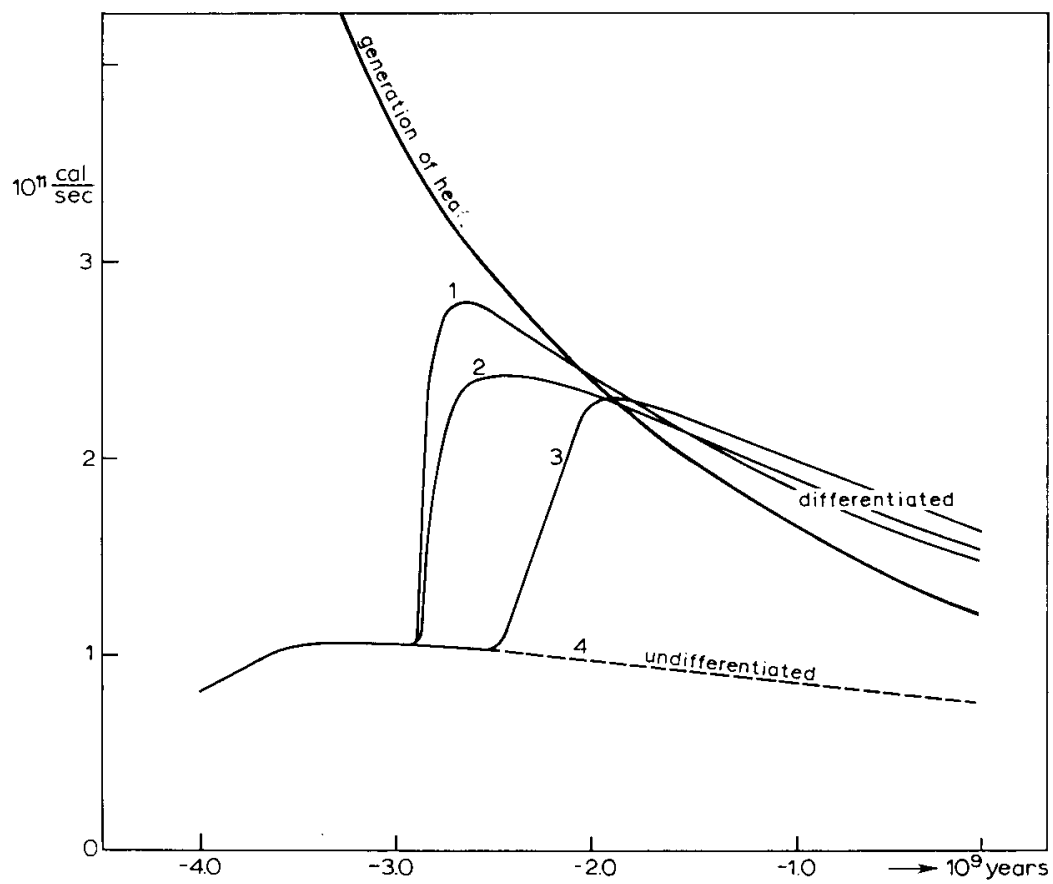

Fig. 9. Moon: time variation of heat generation (thick line) and of total heat loss through the surface. $95 \%$ of radioactive elements from the partially melted interior are transported onto the surface (curve 1), evenly distributed in the outer layer of $50 \mathrm{~km}$ (curve 2), or concentrated at the bottom of the outer solid layer (curve 3). If the Moon remains undifferentiated, the heat loss would follow the dashed curve. In all variants the heat loss is slightly underestimated because heat fluxes for the equatorial zone were extrapolated to the whole surface of the Moon. 
histories of the Earth, Moon and planets for more and more realistic models. (Urey, 1952; MacDonald, 1963; Lubimova, 1958; Majeva, 1964, 1967, 1969, 1971a, b; Reynolds et al., 1966; Lee, 1968; Levin, 1962, 1966b; Levin and Majeva, 1960).

On the basis of a chondritic content of radioactive elements in silicates and a moderately high initial temperature of the order of $1000 \mathrm{~K}$, calculations give plausible thermal histories for all bodies of the terrestrial group.

In Mercury the large content of metallic iron produces a high average heat conductivity while the average content of radioactive elements is probably low. Therefore its interior was never molten and is cooling at the present time. (Majeva, 1969). For the other terrestrial planets and the Moon calculations give more or less molten interiors.

For the Earth, models with an iron core but without convection in the mantle, give nearly complete melting of the latter, contrary to seismic evidence (Majeva, 1971a).

Models with a core of metallized silicates can be easily adjusted to give (1) a correct value of surface heat flow, (2) a solid mantle with temperatures in the upper mantle

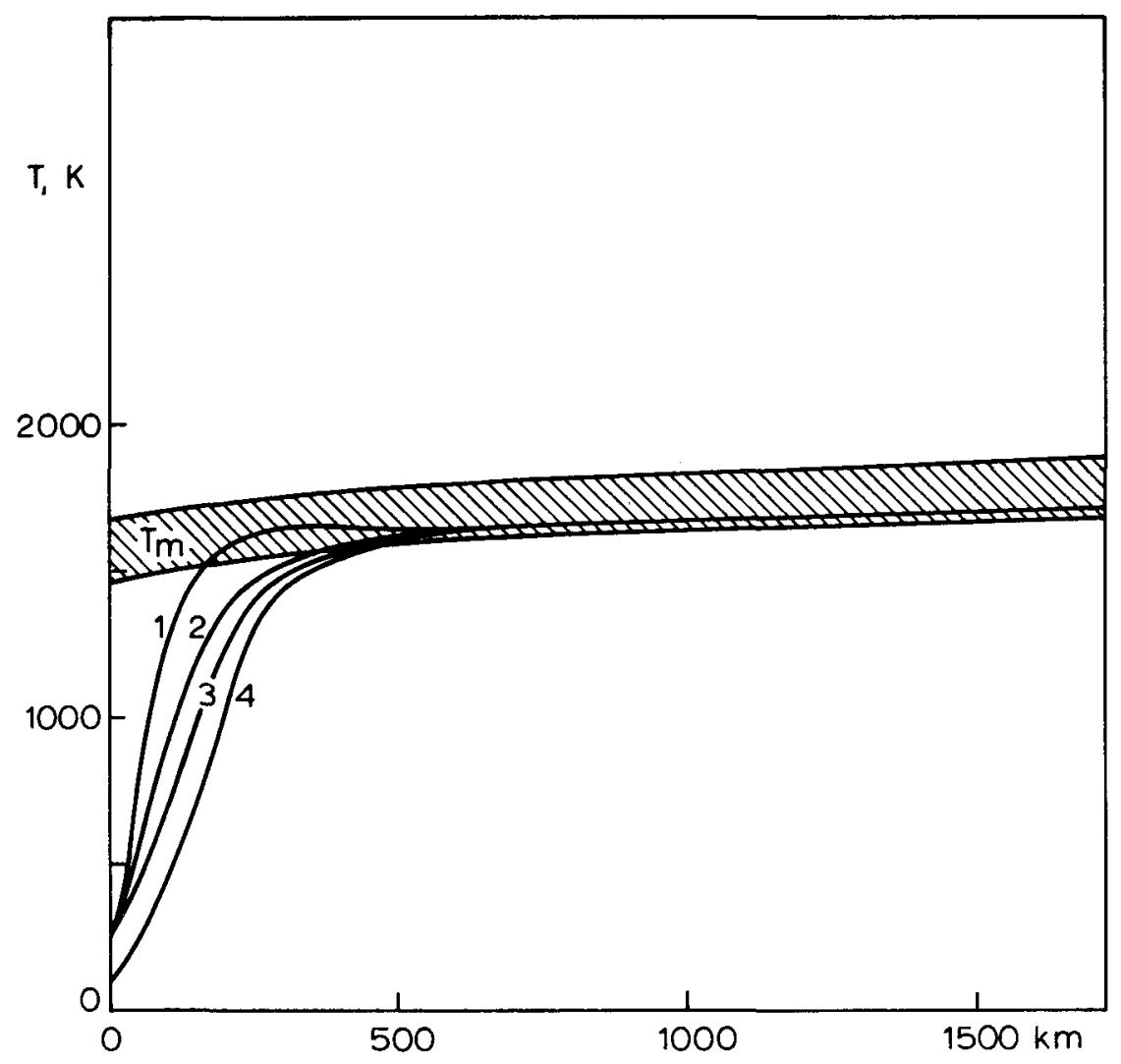

Fig. 10. Moon: Present temperature distribution in the equatorial zone (curves 1-3; see caption, Figure 9) and near the poles (curve 4, redistribution of radioactive elements the same as for curve 2). (For explanation of the hatched zone, see caption to Figure 8.) 
close to melting, (3) a liquid outer core and (4) a superadiabatic heat flow from the core into the mantle. (Majeva, 1967.)

Results for Venus are similar to that for the Earth (Majeva, 1969). A small zone of melting in the upper mantle for the model with a metallized core can be avoided by a small adjustment of thermal parameters. But at the present moment there is no evidence that such avoidance is necessary.

For the Moon the initial heating of its interior changed long ago into cooling, after partial melting of silicates and redistribution of radioactive elements. At present the Moon has an outer solid layer several hundred kilometers thick. (Levin, 1962, 1966b; Majeva, 1971b). Lunar samples brought from maria sites by Apollo missions confirm that the lunar interior underwent melting and differentiation more than 3 billion years ago. The departure of the figure of the Moon from equilibrium and the prcsence of mascons confirm the existence of a thick outer solid layer.

But the brecciated granite-like rock 12013, some parts of which have an age of $4 \frac{1}{2}$ billion years, and similar ages for lunar, dust represent a puzzle. One can only hope that the solution of this puzzle will not destroy the present day ideas of thermal histories for the terrestrial planets.

As a final remark, I would like to stress that at the present time the astronomers studying the terrestrial planets are under increasing pressure from the results obtained by physical chemists, nuclear physicists and petrologists.

We are overloaded by new data that will be extremely important in clarifying the origin of the Earth and planets. But sometimes the interpretation of these data by non-astronomers is in real or apparent conflict with the ideas of astronomers. Closer contacts between scientists of different specialization are becoming more and more important.

\section{References}

Al'tshuler, L. V., Krupnikov, K. K., Lebedev, B. N., Zhuchikhin, V. I., and Brazhnik, M. I: 1958, Zh. Eksperim. Teor. Fiz. 34, 874.

Binder, A. B.: 1969, J. Geophys. Res. 74, 3110.

Birch, F.: 1965, J. Geophys. Res. 70, 6217.

Cameron, A. G. W.: 1962, Icarus 1, 13.

Ganapathy, R., Keays, R. R., Laul, J. C., and Anders, E.: 1970, Geochim. Cosmochim. Acta 34, Suppl. 1, 1117.

Elsasser, W. M.: 1963, in Earth Sci. and Meteoritics (ed. by J. Geiss and E. D. Goldberg), Chapter 1 North-Holland Publ. Co., Amsterdam.

Hanks, T. C. and Anderson, D. L.: 1969, Phys. Earth and Planet Inter. 2, 19.

Kormer, S. B. and Funtikov, A. I.: 1965, Izv. Akad. Nauk SSSR. Fizika Zemli, No. 5, 1.

Kozlovskaya, S. V.: 1962, Voprosi Kosmogoniii 8, 145.

Kozlovskaya, S. V.: 1966, Astron. Zh. 43, 1081.

Kozlovskaya, S. V.: 1969, Astrophys. Letters 4, 1.

Larimer, J. W.: 1967, Geochim. Cosmochim. Acta 31, 1215.

Larimer, G. W. and Anders, E.: 1967, Geochim. Cosmochim. Acta 31, 1239.

Lee, W. H. K.: 1968, Earth Planet. Sci. Lett. 4, 270, 277.

Levin, B. J.: 1962, in The Moon, IAU Symp. No. 14, 157.

Levin, B. J.: 1966a, Proc. Caltech - JPL Lunar Planet. Conf. p. 61; Astron. Zh. 43, 606.

Levin, B. J.: 1966b, in The Nature of the Lunar Surface (ed. by W. H. Hess, D. H. Menzel, and J. A. O'Keefe), Hopkins Press, Baltimore, p. 267. 
Levin, B . J.: 1970, in Surfaces and Interiors of Planets and Satellites, Academic Press, London, Chapter 8.

Levin, B. J. and Majeva, S. V.: 1960, Dokl. Akad. Nauk SSSR 133, 44.

Lubimova, H. A.: 1958, Geophys. J. RAS 1, 115.

Lustikh, E. N.: 1948, Dokl. Akad. Nauk SSSR 59, 1417.

Lyttleton, R. A.: 1963, Proc. Roy. Soc. London, Ser. AA 275, 1.

MacDonald, G. J. F.: 1962, J. Geophys. Res. 67,

MacDonald, C. J. F.: 1963, Space Sci. Rev. $2,473$.

MacDonald, G. J. F. and Knopoff, L.: 1958, Geophys. J. RAS 1, 284.

Majeva, S. V.: 1964, Dokl. Akad. Nauk SSSR 159, 294.

Majeva, S. V.: 1967, Izv. Akad. Nauk SSSR, Fizika Zemli, No. 3, 3.

Majeva, S. V.: 1969, Astrophys. Letters 7, 11.

Majeva, S. V.: 1971a, Izv. Akad. Nauk SSSR, Fizika Zemli, No. 1, 3.

Majeva, S. V.: 1971b, Izv. Akad. Nauk SSSR, Fizika Zemli, No. 3.

McQueen, R. G. and Marsh, S. P.: 1960, J. Appl. Phys. 31, 1253.

Nakamura, Y. and Latham, G. V.: 1969, J. Geophys. Res. 74, 3771.

Plagemann, S.: 1965, J. Geophys. Res. 70, 985.

Reynolds, R. T., Fricker, P. E., and Summer, A. L.: 1966, J. Geophys. Res. 71, 573.

Reynolds, R. T. and Summers, A. L.: 1969, J. Geophys. Res. 74, 2494.

Ringwood, A. E.: 1959, Geochim. Cosmochim. Acta 15, 157.

Ringwood, A. E.: 1966, Geochim. Cosmochim. Acta 30, 41.

Ringwood, A. E.: 1970, Earth Planet. Sci. Lett. 8, 131.

Safronov, V. S.: 1954, Astron. Zh. 31, 499.

Safronov, V. S.: 1958, Voprosy Kosmogonii 6, 63.

Safronov, V. S.: 1965, Izv. Akad. Nauk SSSR, Fiz. Zemli, No. 7, 1.

Safronov, V.S.: 1969, Evolution of the Preplanetary Nebula and the Formation of the Earth and Planets (in Russian), Nauka, Moscow.

Schubert, G., Turcotte, D. L., and Oxburgh, E. R.: 1969, Geophys. J. RAS 18, 441.

Turekian, K. K. and Clark, S. P.: 1969, Earth Planet. Sci. Lett. 6, 346.

Trunin, R. F., Gonshakova, V. I., Simakov, G. V., and Galdin, N. E.: 1965, Izv. Akad.Nauk SSSR, Fiz. Zemli, No. 9, 1.

Uffen, R. J.: 1952, Trans. Amer. Geophys. Union 33, 893.

Urey, H. C.: 1952, The Planets: Their Origin and Development, Yale University Press, New Haven.

Urey, H. C.: 1962, in 'The Moon', IAU Symp. 14, 133.

Zharkov, V. N.: 1960, Dokl. Akad. Nauk SSSR 135, 1378.

Zharkov, V. N.: 1962, Trudy Inst. Fiziki Zemli, No. 20 (187), 3.

Whipple: Thank you. Professor Levin has beautifully presented the paradox that faces this panel.

I now call on Sir Harold Jeffreys who is the most solid Earth man I know.

Jeffreys: I have believed, I think, 4 theories of the origin of the solar system in my time but am not satisfied with any.

The chief evidence for former fluidity of the Earth is: (1) the existence of the land and water hemispheres; (2) the upward concentration of radioactivity. The former cannot be explained if the Earth was completely fluid, or after it was thoroughly solid. But if it passed through a partly fluid state, separated matter could mostly be transported to one side by convection. The second is explained by Goldschmidt's mechanism of fractional separation of the radioactive elements during solidification. No other explanation of either exists.

With the present estimates of about $4.5 \times 10^{9} \mathrm{yr}$ for the age of the Earth, the thermal history needs considerable modification, especially because heat generation by $\mathrm{U}^{235}$ 
and $\mathrm{K}^{40}$ could originally have been much stronger. It is possible that even if the Earth was originally cold and had originally uniform composition melting could take place, in about $10^{9} \mathrm{yr}$. Then the processes decribed above could begin.

Whipple: Thank you. Your vigourous scepticism is characteristic of youth, proving the youthful character of your mind.

Professor S. K. Runcorn directed the panel's attention to two points: (1) the important role of planetary cores, and (2) the treatment of the mechanical and thermal history of the planets. In discussing planetary magnetism, we have to accept that a dynamo effect leads to the presence of planetary magnetic fields. For those planets without rotation, there would be none. Until recently, this fitted for the Earth and Jupiter, while the other planets could be supposed to have small cores or slow rotation. However, the recent findings from Apollo of a lunar magnetic field $3 \times 10^{9} \mathrm{yr}$ ago implies a small contemporary lunar core.

The density of Mars provides good evidence of an iron core, but there is a suggestion, first made by Urey, that all the iron has not yet reached the core. It is necessary to estimate more clearly the sizes of the cores and thus the thermal histories. Years ago, cores were of importance only in the equations of the diffusion of heat, and it was assumed that convection could play a role in a rigid body. From the present boundary conditions, one could work back in time. Now, the discussion of mechanical and thermal properties are more difficult for planets than for stars, as the knowledge of the interior depends on still rudimentary solid-state physics.

Plate tectonics imply thermal convection and solid creep. The dependence on temperature adds to the complexity of the problem.

Reynolds: Sir Harold Jeffreys, from his vast store of wisdom and experience, has expressed a quite healthy scepticism regarding the state of our current knowledge of the internal constitution and thermal history of the planets. Much of the knowledge that we have, or think we have, about these subjects comes from the calculation and analysis of mathematical models, constructed with parameters and boundary conditions determined as accurately as possible from astronomical and geophyscial data. Such models can serve as a vital element in the process of increasing our understanding of the planets, provided that the interpretations of both the values and limitations of these models are maintained in a proper perspective.

Detailed thermal history models have been calculated by such prominent investigators as Urey, Lubimova, MacDonald, and Levin; as well as by many others. The equation of heat conduction for a spherically symmetric body with internally distributed heat sources was solved for a variety of initial and boundary conditions. These authors generally concluded that conditions for melting could be reached for large regions of the interiors of the terrestrial planets. More realistic models were therefore constructed which included such effects of melting as the energy required for the latent heat of fusion and fluid convection within a molten region. Processes which concentrate the heat producing radioactive isotopes preferentially within certain regions have occurred within the Earth, Moon, and meteorites; such processes must be considered in a study of the thermal history of a planetary body. The movement of 
radioactive heat sources towards the surface is a very effective method of heat transfer since it results in the removal, not only of the excess specific heat, but also of all the energy subsequently released by radioactive decay.

I might make a few brief remarks on the particular subject of the thermal history of the Moon that have arisen from our thermal model calculations. Evidence has accumulated from studies of the returned lunar samples that some surface areas of the Moon were formed roughly 4.5 to $4.7 \times 10^{9} \mathrm{yr}$ ago and that extensive basaltic lava flows covered some of the mare regions about 3.4 to $3.8 \times 10^{9} \mathrm{yr}$ ago.

Model calculations, starting with either a low temperature undifferentiated moon or a completely molten and differentiated moon, have difficulty in providing conditions for melting at the experimentally determined ages. A two-stage melting process, which includes an outer layer initially melted by accretional heating combined with a later period of rising temperatures in the outer layers due to the differentiation of radioactive heat sources within the lunar interior, can produce a model which is consistent with the observations. Such a model involves many assumptions and choices of physical parameters which cannot be discussed here.

The thermal history depends critically upon the choice of initial conditions and the thermal history calculations are thus inextricably linked with theories of origin for both the Moon and the solar system. At present great uncertainties exist in all of the many theories of the origin of the solar system. There are, however, encouraging prospects for a considerable reduction of these uncertainties in the near future. Further studies of the Apollo 11 and 12 experiments and planned future spacecraft missions to the Moon and planets will continue to return significant quantities of new information. A combination of new experimental and observational constraints with improved theoretical modeling techniques should permit us to reverse the trend towards an increasing proliferation of models and theories and perhaps to finally begin to gain a real understanding of the origin and subsequent evolution of the solar system.

Kopal: I should like to use this opportunity for making two comments: one concerning the introductory address by Dr Levin to this part of our session; and the other concerning the more general issues raised in the earlier part of this morning.

With regard to the problem of the internal temperature of the lunar globe, I recall that on a previous occasion when we met to discuss the same problem at Goddard five years ago, Professor Urey cautioned Dr Levin 'not to melt the Moon'; and, in Urey's absence, I should like to raise the same voice of caution today. I am glad to learn from his address that Levin agrees now the Moon to be solid (and, to this extent, 'cold') down to a depth of several hundred kilometers;* for what is below that depth is largely hypothetical (we possess as yet no observational evidence bearing directly on the subject), and also largely irrelevant to the phenomena which we can

* Note in proof by Levin: This is a misunderstanding. In 1965 I reported the same model of lunar thermal history and predicted the existence of a solid outer layer a few hundred km thick (Levin, 1966b). Only calculations reported in 1965 were made with a less elaborate programme for a electronic computer. 
observe on the lunar surface. For surely even those who regard the lunar maria to be solidified lava pools do not seek the origin of this lava so deep below the surface what could pump it up through a solid shell several hundred kilometers thick? Whether or not the Moon possesses a small core in which the internal temperature has exceeded the melting point of rocks (at prevailing pressure) constitutes a question which is still largely hypothetical (depending on the amount of radioactivity which we assume to be operative in the interior), and without any direct relevance to the phenomena which can be observed on the surface of our satellite.

The second comment which I should like to make at this opportunity refers to Dr. Herbig's remarks which we heard earlier this morning. I should like to applaud his attitude that, in problems as complicated as that of the origin - not only of the Moon, but of the solar system as a whole - we should seek whenever possible a guidance from the observations. Now, in this connection, I should like to mention that we know in the sky at least one object (at a distance of a mere 1300 parsec away from us) which may give us certain valuable clues in this connection. This is the well-known eclipsing system of epsilon Aurigae, whose secondary (eclipsing) component apparently possesses the form of a flat disc, which is semi-transparent and dims light passing through it non-selectively; therefore, it consists of particles which are large in comparison with the wavelength of observations. The equatorial dimension of this disc appears to be of the order of $40 \mathrm{AU}$ and its mean temperature, about $500 \mathrm{~K}$.

This model of the secondary component of $\varepsilon$ Aur, constructed largely on the basis of facts supplied directly be the observations, bears a rather striking resemblance to the models of the 'primitive solar nebula' from which our planetary system is supposed to have originated, and about which we heard so much earlier this morning. There are, however, also some obvious differences. First, the total mass of the disk-like component of $\varepsilon$ Aur appears to be not less than 20 solar masses - the system is a supergiant one, whose principal component of spectral class F2 I cannot be much more than a million years old - and, therefore, its secondary component may be just now (astronomically speaking) giving birth to a planetary system very much more massive than our own. Secondly, its disk-like structure emits virtually no light of its own (apart from a small trickle in the deep infrared, which fixes its temperature to some $500 \mathrm{~K})$; and, therefore, it would vote for a 'cold' planetary nebula in this sense rather than a 'hot' one.

Whipple: Professor Kopal, thank you. I think that Professor Kopal has cheated a bit by bringing a new problem that may be more difficult than the one we set out to solve.

Anders: I am afraid I must disagree with my good friend, Professor Levin, concerning the composition of the inner planets. Professor Levin has shown, quite ingeniously, that the density differences can be interpreted in terms of metallized silicates rather than variations in iron content. This way all inner planets, except Mercury, can have essentially the same bulk composition.

But actually there is good evidence for chemical fractionations in the solar nebula 
which separated metal, silicates, volatiles, and highly-refractory compounds from each other. This evidence comes in part from meteorites and in part from the Earth and Moon.

Among the 5 classes of chondrites, a number of refractory elements such as Ca vary by a factor of 2 (Larimer and Anders, 1970), volatiles such as K by factors of 2 to 1000 (Larimer and Anders, 1967), and various elements that are easily reduced to metal such as Fe by a factor of 3 (Urey and Craig, 1953). All together, some 50-odd elements are thus fractionated. It is particularly remarkable that these elements are depleted by constant factors. This is not at all what one would expect for igneous differentiation in a meteorite parent body, and for this and other reasons the prevailing view is that these fractionations happened in the solar nebula.

It seems that 3 kinds of fractionation took place, involving loss of refractory elements, volatiles, and metal phase. It is not hard to find mechanisms for such fractionations. Refractory elements would be enriched in an early condensate (or in a volatilization residue) and might separate from the gas by settling toward the median plane. Metal grains might become separated from silicate grains owing to their ferro-magnetism (Wood, 1963; Harris and Tozer, 1967). Indeed, there is evidence from trace element data that the fractionation happened close to the Curie point of nickel-iron, $900 \mathrm{~K}$ (Larimer and Anders, 1970). Volatiles, in turn, would stay behind in the gas and condense only gradually, as temperatures fell.

It seems that the Earth and Moon experienced the same fractionation processes, but to a different degree. Of course, it is hard to estimate the bulk composition of a planet of which only the outermost $0.1 \%$ is accessible to us. We must limit ourselves to elements that are largely concentrated in the crust. Such comparisons have been made for volatile elements $(\mathrm{Rb}, \mathrm{K})$ and refractory elements $(\mathrm{Sr}, \mathrm{U})$. Since three of these four elements are radioactive, the abundance arguments can be checked by isotopic and heat balance arguments. The conclusion is that the Earth is depleted in volatiles and enriched in refractories, by an overall factor of 5 or so, relative to ordinary chondrites (Gast, 1960; Wasserburg et al., 1964). For the Moon, the fractionation is still more extreme, by a factor of 25 to 30 . And it has been suggested on petrologic grounds that material of the composition of lunar basalts cannot be derived from chondritic matter, but requires a parent material enriched in refractories (Ringwood and Essene, 1970).

Thus there are significant differences in the abundance of refractories and volatiles in the Earth, Moon, and chondrites. There are similar differences in the iron content of chondrites, and it is tempting to explain the density differences among planets by the same mechanism. The choice before us thus is whether to extend the fractionation processes in chondrites to the planets, or whether to assume a new and different mechanism for the planets, e.g. metallized silicates. In the latter case, we must also explain how the planets escaped the metal-silicate fractionation seen in chondrites and, as conceded by Prof. Levin, in Mercury.

Such explanations can of course be found; for example, one can postulate that the temperature in the region of the inner planets was above the Curie point of nickel-iron. 
But I think it is fair to say that metallized silicates, instead of simplifiying the picture, make it more complicated by requiring a greater number of assumptions.

\section{References}

Gast, P. W.: 1960, 'Limitations on the Composition of the Upper Mantle', J. Geophys. Res. 65, 1287. Harris, P. G. and Tozer, D. C.: 1967, 'Fractionation of Iron in the Solar System', Nature $215,1449$. Larimer, J. W. and Anders, E. : 1967, 'Chemical Fractionations in Meteorites II: Abundance Patterns and Their Interpretation', Geochim. Cosmochim. Acta 31, 1239.

Larimer, J. W. and Anders, E.: 1970, 'Chemical Fractionations in Meteorites. III. Major Element Fractionations in Chondrites', Geochim. Cosmochim. Acta 34, 367.

Ringwood, A. E. and Essene, E.: 1970, 'Petrogenesis of Lunar Basalts and the Internal Constitution and Origin of the Moon', Science 167, 607.

Urey, H. C. and Craig, H.: 1953, 'The Composition of Stone Meteorites and the Origin of the Meteorites', Geochim. Cosmochim. Acta 4, 36.

Wasserburg, G. J., MacDonald, G. J. F., Hoyle, F., and Fowlet, W. A.: 1964, 'Relative Contributions of Uranium, Thorium and Potassium to Heat Production in the Earth', Science 143, 465.

Wood, J. A.: 1963, 'On the Origin of Chondrules and Chondrites', Icarus 2, 152.

Gratton: I did not hear any mention of the rotation of planets. Is that due to the fact that no information can be obtained from this side or to the fact that it is an exceedingly difficult problem? It seems time that this problem ought to receive more attention.

Hoyle: The case of Uranus shows that the problem of planetary rotations is not straightforward. The fact that Uranus spins about an axis lying close to the ecliptic suggests that two approximately comparable bodies coalesced to form this planet, and that it was the manner of coalescence that determined the subsequent spin.

The situation would be easier to calculate for the planet aggregating either from a gas or a swarm of small particles, but until the two possible modes of formation - a few comparatively large bodies falling together, or aggregation from a swarm - have been disentangled the situation remains obscure.

Gold: About the spins of the planets, I said this morning that I thought one had to have an early period in the solar system where bodies formed and recollided. One argument for that is the erratic nature of the spins. From the statistical distributions of the angular momenta of the planets we have to single out those that have not lost angular momenta to tidal friction. From the then remaining scatter of the angular momenta of the planets, one can make a statistical estimate of the proportion of the material that had formed together in large pieces, assuming almost random distribution of angular momentum, to the proportion of the material that fell together in small stuff.

About $\frac{3}{4}$ of the planetary masses must have come together in quite large chunks, certifying that one did have planetesimals before and they must have had a career of colliding bodies.

Whipple: A very important observation. A remnant of comets formed near the plane of the planets beyond Neptune might have contributed to the mass calculated for Pluto. I have asked Duncombe to look into the matter. 
Safronov: In order to avoid difficulties with the formation of the Earth's iron core some authors try to obtain a hot initial state of the Earth supposing that it has formed during a very short time $-10^{5}-10^{6} \mathrm{yr}$. Such supposition is however quite inadmissible. The rate of accumulation of the Earth depended on the relative velocities of bodies. The velocities were determined in turn by gravitational perturbations of bodies and can be found if the mass distribution of the bodies is known. Estimates show that the time for the Earth's formation was about $10^{8} \mathrm{yr}$ and not less than $10^{7} \mathrm{yr}$, i.e. two or three orders of magnitude greater than it is assumed by Hanks and Anderson, and Ringwood. Various additional forces (magnetic and electrical ones, resistance of a gas) could considerably accelerate the growth of small particles. But they did not change the rate of growth of large bodies and could not appreciably decrease the whole period of growth of the planets most of which was when these bodies became large (more than $10^{2} \mathrm{~km}$ ).

Important sources of heating of the Earth were impacts of big bodies of asteroidal sizes. They could produce appreciable large-scale inhomogeneities of temperature. The temperature of excessively heated regions might be near to the melting point. It is not excluded that the formation of continents might be connected with the existence of such regions.

$O$ 'Keefe: On the accumulation times, any statement that an accumulation in time shorter than $x$ yr is impossible has certain assumptions in mind and it is important to emphasize the tremendous number of processes that, I do not believe that Dr Safronov has taken into consideration and that are possible in a gaseous nebula. There will be some pressure gradient in the radial direction in the disc that will cause friction between the gas and the particles in the sense that the gas receives a partial hydrostatic support. The particles are in free orbital motion and therefore revolve at a slightly different rate. Therefore particles can spiral inwards.

Turbulence in the presence of radioactivity necessarily leads to charge - separation and the building up of an electric field. One might expect that the energy of the field would tend to approach the turbulent energy density.

The primitive nebula must be extremely complicated and complicated processes can take place; for example, small-particle charges can cause impacts, lightning can cause fusion and so on. In the larger size-range, the gas motions tend to give the massive bodies different trajectories from the smaller bodies. One cannot really state that there is a definite lower limit to the accumulation time, in view of such uncertainty, for a body like the Earth.

Regarding temperature, major collisions responsible for the final state of bodies like the Earth, interior temperatures of the order of $10^{4} \mathrm{~K}$ follow automatically, and therefore not only must the planet be largely liquid but also layers of silicates may even be gaseous.

The size of the nebula, the mass, depends on the early history of the angular momentum. Observationally, in the T Tauri stage, several tenths of a solar mass are lost directly from the central star.

It does not seem difficult that comparable amounts of mass should be picked up 
from the weakly-bound surrounding solar nebula and carried off with the T Tauri solar wind into space.

Brecher: Yet another approach to the origin of the solar system which seems to be warranted by observations in the infrared and by studies of lunar samples and meteoritic composition. The condensation mechanism proposed takes place from a low density plasma-gas mixture which is at a much higher temperature than the solid grains condensing from it and fed by it. This is an extreme thermal dis-equilibrium situation between the solid grains and the gas-plasma phase. It seems to be more justified than assumptions of thermo-chemical equilibrium at condensations that are assumed in most theories. The controlling factors are specializations of elements according to the ionization potential, and on that basis we seem to be able to explain the abundance of noble elements in iron meteorites, the mercury anomaly and other volatile abundances that have had difficulties in other theories. We are concentrating on iron-nickel condensation at low temperature and we seem to be able to grow perfect crystalline phases with both alpha and gamma phase, used as a thermometer for the formation of meteorites until now. We do not seem to need a complicated thermal history, assuming cooling from high temperature to the range $300-400 \mathrm{~K}$, in order to explain the phases observed in crystalline iron meteorites.

Experiments by Meyer and Arrhenius have shown chemical fractionation patterns from a cosmic mixture involving hydrogen, oxygen, $\mathrm{Mg}, \mathrm{Ca}$, and $\mathrm{Al}$, and crystallines very similar to observed silicates eg. olivines, were condensed at quite low temperature $\approx 600 \mathrm{~K}$.

The recondensation from a gas phase may be a primordial process whose traces can be deciphered in meteoritic and lunar samples.

A metal core is not really needed to explain the magnetic moments in meteorites since the chemistry and position of remanent magnetisation may be fossils from the accretionary processes of grains and could account for such magnetic moments. In geophysics, they have been mistaken many times for thermal remanent magnetization.

Fission tracks in chondritic material seem to be isotropically distributed on grain surfaces, implying that the grains were individually suspended in free space for quite some time during the accretion of their meteoritic body, confirming the jet-stream stage in Professor Alfvén's theory of focussing the grains into streams where the relative velocity decreases to a point which allows the accretion process to dominate over collision fragmentation processes.

Hoyle: Regarding the original planetary mass, this morning I set the mass as low as one can reasonably do. Safronov wants to increase it by a factor of 5 and Cameron would go another power of 10 . If the original mass is set very high, then one must have sone powerful process for getting rid of the large excess. One can then ask what would have happened if the process had been just a little stronger. This is not a decisive argument against starting with a large mass because we do not know that we are looking at a typical case. Only in those cases where a little bit is left could you get creatures that could argue about this problem. You can argue, in these large mass cases, that we are living in a freak example where just a little bit has been left. That would make all problems hard but it could be right. 
Pellas: What have Professor Alfvén and his group obtained concerning the mineral phases synthesized at low temperature in the plasma? If there are some phases not present in meteorites, there is an objection to the model.

Two years ago, I was the first to propose an irradiation in space on crystals after condensation. I do not now think this is the real way to explain the irradiation. I think it took place on the surface of asteroidal bodies on which gravity is low and on which shocks are numerous and produce some way of irradiating the crystals all around. The remarks of Mrs Brecher do not give a proof of irradiation in space. 